\title{
Studies of waves in sunspots using spectropolarimetric observations
}

\author{
Gordon A. MacDonald ${ }^{1}$ and S. P. Rajaguru ${ }^{2}$ \\ ${ }^{1}$ California State University, Northridge, \\ 18111 Nordhoff St., Northridge, CA 91330, USA \\ email: gordon.macdonald.31@my.csun.edu \\ ${ }^{2}$ Indian Institute of Astrophysics, \\ II Block, Koramangala, Bangalore 560 034, INDIA \\ email: rajaguru@iiap.res.in
}

\begin{abstract}
We observe the acoustic velocity oscillations in and near active region NOAA 10960 on 8 June, 2007 using observations from the IBIS instrument at the Dunn Solar Telescope at NSO/Sacramento Peak and simultaneous Hinode BFI/SP data. Inversions were performed on the spectropolarimetric datasets in order to get magnetic field information for the AR. A time series of Doppler maps from line bisectors and Stokes $V$ zero-crossing was constructed and allowed us to construct power maps for the AR. Past works by various authors have shown that acoustic power in the solar atmosphere is strongly influenced by magnetic field strength and inclination. Our study also explores this, but in addition, we also discuss the role of oscillations due to purely magnetized gas in the photosphere. Our preliminary results for this study are presented.
\end{abstract}

Keywords. Local helieseismology, active regions

\section{Introduction}

It was shown by Hindman \& Brown (1998) that acoustic oscillations within sunspots are strongly influenced by magnetic field strength. Later, Nicolas et al. (2005) demonstrated that the amount of influence cannot be solely determined by the photospheric magnetic field strength and Schunker \& Braun (2010) showed that the acoustic power is strongly influenced by magnetic field inclination. We attempt to demonstrate how the magnetic field of AR's change the oscillatory behavior of the solar atmosphere.

Our datum are observations of NOAA 10960 on 8 June, 2007 at S07W17 from the IBIS instrument located at the Dunn Solar Telescope at Sacramento Peak. The IBIS data observes simultaneous Stokes $I, Q, U, V$ parameters in the magnetic line FeI $6173 \AA$ and also continuum intensity in the non-magnetic line FeI $7090 \AA$. Our set has a cadence of 47.5 seconds and a total observation time of 7.5 hours.

Using these data, we find the time series of the line bisector and also the Stokes $V$ zero crossing and extract the oscillation frequencies by performing a FFT. It will also be possible to obtain a time series of intensity variations using the CaII $\mathrm{H}$ images. The zero crossing Doppler shift will be of particular interest since it gives the Doppler speed of only the magnetized portion of the gas. Inversions were performed on the IBIS $6173 \AA$ line to extract information concerning the local magnetic field. 


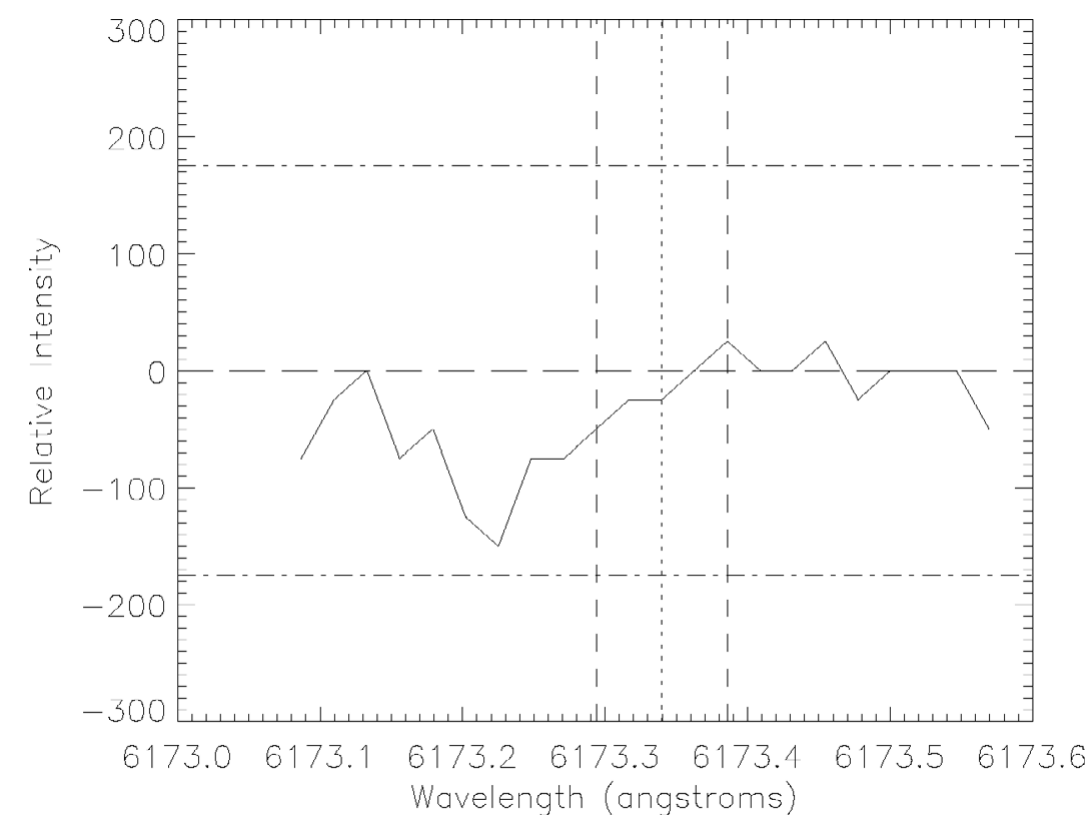

Figure 1. Seen here is a single-lobed profile which is created by the weak fields normally found in the quiet sun Sigwarth (2001). The two horizontal dashed lines indicate the cutoff intensity of \pm 175 and the vertical dashed lines indicate the $\pm 45 \mathrm{~m} \AA$ interval where the $\mathrm{ZC}$ is expected to be found. This is an example of a profile that would not be analyzed, as the amplitude of each lobe is far too small to be considered for analysis. In addition, the fact that there exists only one lobe excludes it from analysis. All profiles which do not meet this criteria are assigned a Doppler speed of 0 .

\section{Data reduction and analysis}

Our study begins with the creation of Doppler maps obtained from FeI $6173 \AA$ line core, wings and Stokes $\mathrm{V}$ zero-crossing (ZC). Stokes $V$ were analyzed based on their lobe amplitude. A 582 element-long time series of these maps is thus obtained, and by performing a FFT on these maps, we are able to get the power spectrum of each pixel in the FOV. By binning these spectra, we may construct power maps for the AR. The intervals used in this study are $2-3 \mathrm{mHz}, 3-4 \mathrm{mHz}$ and $4-6 \mathrm{mHz}$.

\section{Findings}

The obvious continuation of quiet region oscillations in the penumbra as seen in the line core may be explained by the field lines being nearly transverse to the LOS. Indeed, this is reflected in the power correlations in Figure 3 where we see a rise in acoustic power in line wings between approximately $50^{\circ}$ and $65^{\circ}$ and a corresponding drop in ZC acoustic power. Horizontal fields seem to suppress these ZC oscillations and enhance those seen in the line wing. Conversely, we see a node-like structure in the Doppler power maps but not in the ZC power maps. In fact, the umbral acoustic power in the $\mathrm{ZC}$ is more than twice as large as the acoustic power viewed in the wings. This is the site of very strong, nearly-vertical magnetic field. Sites such as these appear to play host to much higher power in $\mathrm{ZC}$ oscillations. The contributions of these magnetic os- 

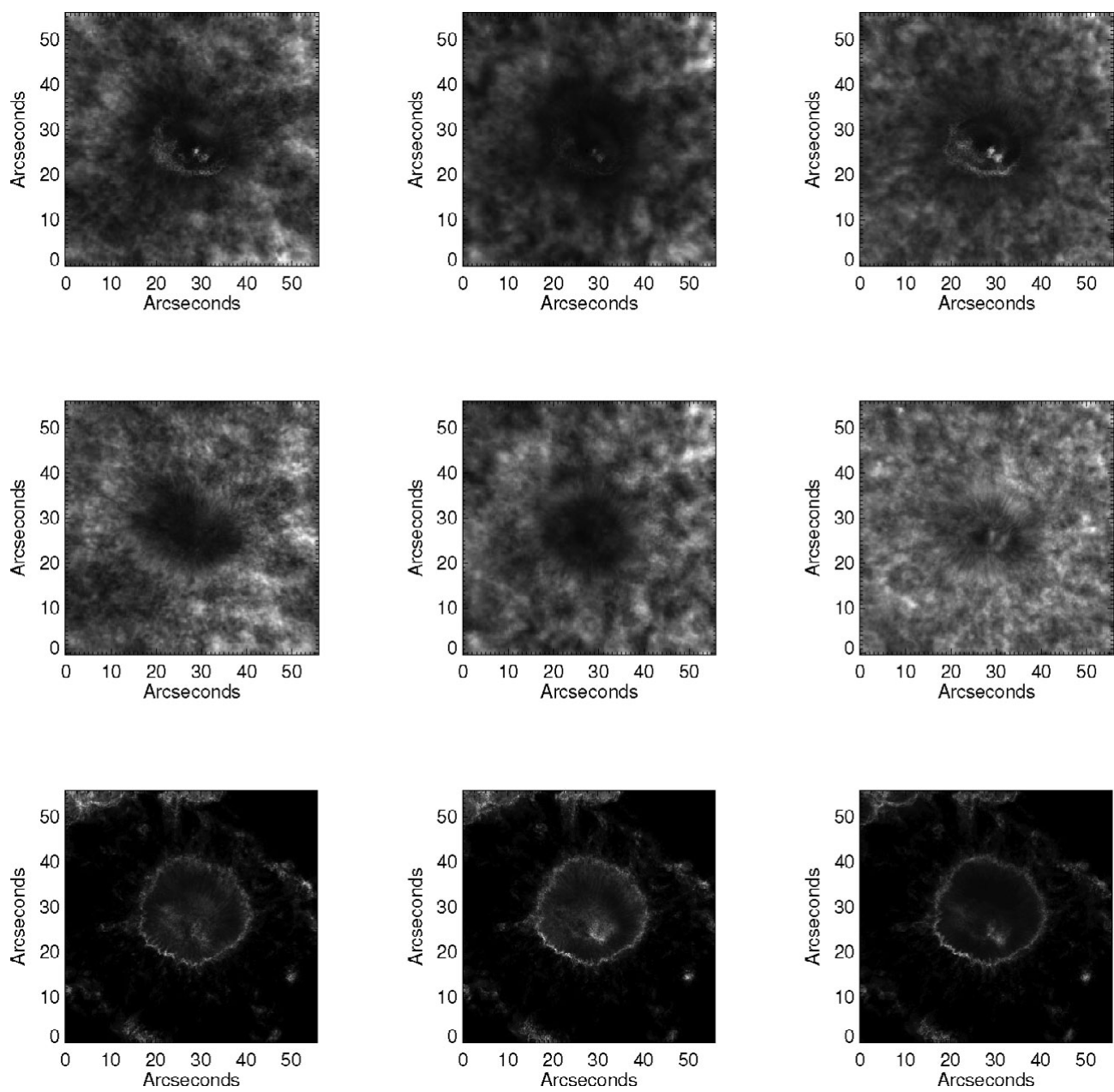

Figure 2. From top to bottom row: power maps for FeI $6173 \AA$ line wing, core and ZC. From left to right column: power spectra for frequency bands $2-3 \mathrm{mHz}, 3-4 \mathrm{mHz}$ and $4-6 \mathrm{mHz}$. We observe umbral node-like structures in the power maps constructed from line wing and core. A thin halo of enhanced power is observed around the outer penumbra in power maps from Stokes $V$ ZC. In each set of three power maps, the units are not scaled and are purely arbitrary.

cillations may simply be greater when strong fields are nearly parallel or anti-parallel to the LOS while those seen in the line core and wings are suppressed by these same fields.

We also observe the localized regions of $\mathrm{ZC}$ acoustic power in the umbra and also an acoustic halo surrounding the outer penumbra. We believe the regions of enhanced oscillations in the umbra to be caused by strong fields in those regions, which give rise to high concentrations of dynamic Stokes $V$ profiles. Since these profiles have 3 ZC's, obtaining an accurate time series of ZC velocities is not trivial and we see that if they are not treated carefully, one may end up with false regions of high-power oscillations. In the case of the halo surrounding the outer penumbra, this may be caused by one of two things: $i$ ) high power oscillations in the magnetic field itself in sites where the field lines dive back into the surface or $i i$ ) the disappearance and reappearance of 

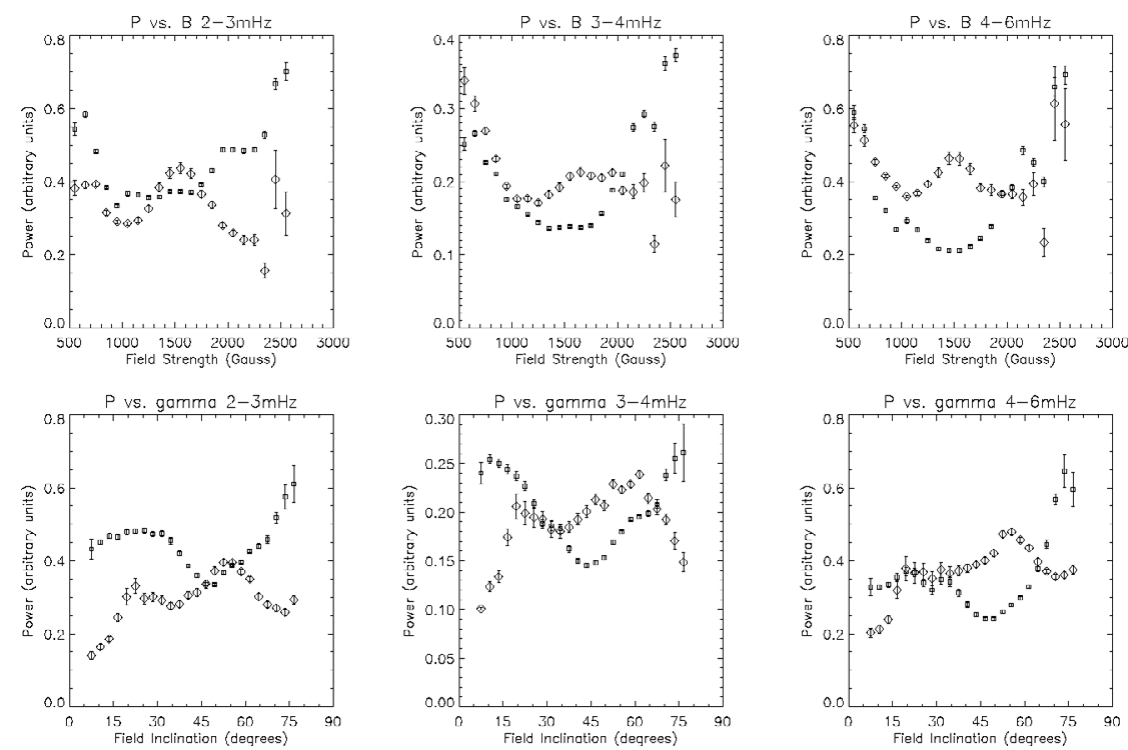

Figure 3. Masked power vs. field strength and inclination for oscillations seen in FeI $6173 \AA$ wings and ZC. The acoustic power (vertical axis) is measured relative to the average power observed in the line wings in the quiet sun in each respective frequency band. Line wing points are denoted by a $\diamond$ and ZC points by a $\square$. We see vastly different behavior of line wing and ZC as we traverse the cross-section of the AR. Error bars represent the standard error, $\sigma / \sqrt{N}$.

intermediate strength Stokes $V$ profiles due to scattering and other seeing effects. Indeed, the former is plausible, as there may previously undetected effects caused by the interface between granular convection and Evershed flows. However, currently the latter is more obvious as we do not see the size or shape of this halo change when we observe in different bands. Furthermore, power in this halo does not change with the frequency band chosen. Thus, the halo may be a consequence of our selection criteria for the ZC analysis.

\section{Acknowledgements}

This work was funded by the National Science Foundation's Office of International Science and Education, Grant Number 0854436: International Research Experience for Students, and managed by the National Solar Observatory's Global Oscillation Network. Our sincere thanks also goes to the academic faculty and support staff at the Indian Institute of Astrophysics. We would also like to thank K. Sankarasubramanian for his counsel on case difficulties encountered during this study.

\section{References}

B. W. Hindman \& T. M. Brown 1998, Astrophys. J., 504, 1029

K. Nagashima, T. Sekii, A. G. Kosovichev, H. Shibahashi, S. Tsuneta, K. Ichimoto, Y. Katsukawa, B. Lites, S. Nagata, T. Shimizu, R. A. Shine, Y. Suematsu,

T. D. Tarbell, \& A. M. Title, 2007, Pub. Astron. Soc. Jap., 59, 631

C. J. Nicholas, M. J. Thompson \& S. P. Rajaguru, 2005, Solar Phys., 225, 213 
A. A. Norton, J. P. Graham, R. K. Ulrich, J. Schou, S. Tomczyk, Y. Liu, B. W. Lites, A. López Ariste, R. I. Bush, H. Socas-Navarro, \& P. H. Scherrer, 2006, Solar Phys., 239, 69

S. P. Rajaguru, R. Wachter, K. Sankarsubramanian, \& S. Couvidat, 2010, Astrophys. J., 721, L86

H. Schunker \& D. .C. Braun, 2010, Solar Phys., DOI 10.1007/s11207-010-9550-3

M. Sigwarth, 2001, Astrophys. J., 563, 1031 